\title{
cha CÍRCULO
}

de

lingüistica
aplicada a la

aplicada a la
comunica

ción

$68 / 2016$

UNA NOTA SOBRE EL MORFO CERO:

EL IMPERATIVO Y LA VOCAL TEMÁTICA

\author{
Antonio Fábregas \\ Universidad de Tromsø-Universidad Ártica de Noruega \\ antonio.fabregas en uit.no
}

Resumen

La noción de afijo cero resulta sumamente polémica en los estudios morfológicos desde Nida (1948), por lo que numerosos autores han propuesto que no pueden emplearse como objetos del análisis morfológico (p. ej., Scalise 1983). Este trabajo proporciona un argumento del español a favor de admitirlos como elementos posibles de la estructura interna de las palabras: sin postular un sufijo cero para la tercera persona singular no es posible dar cuenta de la distribución de formas como sal (imperativo) frente a sal-e (presente de indicativo).

Palabras clave: morfo cero, vocal temática, imperativo, presente, flexión verbal

Fábregas, Antonio. 2016.

Una nota sobre el morfo cero: el imperativo y la vocal temática.

Círculo de Lingüística Aplicada a la Comunicación 68, 100-116.

http://www.ucm.es/info/circulo/no68/fabregas.pdf

http://revistas.ucm.es/index.php/CLAC

http://dx.doi.org/10.5209/CLAC.54523

C 2016 Antonio Fábregas

Círculo de Lingüística Aplicada a la Comunicación (clac)

Universidad Complutense de Madrid. ISSN 1576-4737. http://www.ucm.es/info/circulo 


\begin{abstract}
A note on zero morphs: imperatives and theme vowels. The notion of 'zero morph' has been extremely controversial in morphological studies since Nida (1948), with the consequence that many authors have argued that such objects cannot be used as ingredients in morphological analysis (for instance, Scalise 1983). This article offers an argument from Spanish that supports their existence: without a zero morph corresponding to the third person singular inflection it becomes impossible to account for the distribution of forms like sal 'come out, imperative', vs. sal-e 'comes, indicative present'.
\end{abstract}

Keywords: zero morph, theme vowel, imperative, present, verbal inflection

\title{
Resumé
}

Une note sur le morphème zéro: l'impératif et la voyelle thématique. La notion de 'morphème zéro' est très controversielle dans les études morphologiques à partir de Nida (1948), de sorte que de nombreux auteurs ont proposé que ceux morphèmes ne peuvent pas être utilisés comme objets d'analyse morphologique (p. ex., Scalise 1983). Cet article fournit un argument de l'espagnol en faveur de les admettre comme éléments possibles de la structure interne des mots: sans postuler un suffixe zéro pour la troisième personne du singulier il n'est pas possible de tenir compte de la distribution dela forme sal (impératif) par rapport à sal-e (indicatif présent).

Mots-clés: morphème zéro, voyelle thématique, impératif, présent, flexion verbale

Índice

1. Los orígenes del rechazo a los morfos cero 102

2. La vocal temática en el imperativo 106

3. Los imperativos 108

4. El presente de indicativo 110

4. Conclusiones 114

Referencias 114 
1. Los orígenes del rechazo a los morfos cero

La ciencia se encuentra a menudo influida por tendencias globales en las que la imposibilidad de aceptar un análisis de un tipo para un caso concreto puede llegar a interpretarse como una negación de que el modo analítico utilizado en ese caso debe rechazarse para todos los casos. Tal es lo que sucede, en nuestra opinión, con los morfos cero en el análisis morfológico. Como es bien sabido, un morfo cero es la representación de un morfema en el que se identifican rasgos morfosintácticos activos en la estructura a los que no se asocia ninguna representación morfofonológica. Si la representación de (1) podría corresponder a un morfema clásico en el sentido de Bloomfield (1933), la representación de (2) correspondería a un morfo cero.

$$
\begin{array}{lll}
\text { REPRESENTACIÓN MORFOSINTÁCTICA: } & \text { [plural] } \\
\text { REPRESENTACIÓN MORFOFONOLÓGICA: } & \text { /-s/ } \\
\text { REPRESENTACIÓN MORFOSINTÁCTICA: } & \text { [plural] } \\
\text { REPRESENTACIÓN MORFOFONOLÓGICA: _ } &
\end{array}
$$

Es bien sabido que durante los años cuarenta, el llamado análisis morfémico usaba morfos cero con cierta libertad. Un ejemplo paradigmático de esto lo encontramos en Hockett (1947), que partía del conocido caso del pasado del verbo go 'ir' en inglés, went. En su propuesta, la representación correcta de esta forma era la de (3), que es perfectamente paralela a la representación del pasado para un verbo regular (4).

$$
\begin{array}{lr}
{\left[[\text { went }]_{\mathrm{V}}\right.} & \varnothing]_{\text {Pasado }} \\
{\left[[\text { learn }]_{\mathrm{V}}\right.} & \text { ed }]_{\text {Pasado }}
\end{array}
$$

En esta teoría, la forma de (3) contiene una variedad morfofonológica del exponente de [pasado], por lo que la manifestación cero no es más que un alomorfo del morfema que normalmente se representa como -ed -lo cual explica que en puridad se hable de morfos cero y no de morfemas cero-. Esta variante quedaría seleccionada por algunas bases verbales, a las que go pertenecería. Una vez insertado el morfo cero, la base verbal sufriría a su vez un proceso de reajuste, en presencia del morfo cero, que haría que la forma go fuera sustituida por su alomorfo went, cuya distribución estaría restringida solo a contextos de pasado.

Como también es conocido, este análisis fue inmediatamente criticado por Nida (1948). Nida observaba que este análisis era sumamente contraintuitivo desde una perspectiva 
psicológica y de adquisición. El análisis implicaba tratar como semánticamente no significativa la alternancia que percibían directamente los hablantes: la alternancia entre go y went, que en la superficie es el cambio entre las formas, no codifica [pasado], sino que es una alternancia meramente morfofonológica que no afecta al significado. A su vez, el elemento que sí producía un cambio de significado no tenía representación en la superficie: el morfo cero. Para Nida, esto era una forma de analizar la alternancia que no podía capturar de ninguna manera la forma en que los morfemas se empleaban, esencialmente, como signos para comunicar distintas nociones.

¿Cuál era, entonces, la propuesta de Nida? Su propuesta fue que las alternancias gramaticales podían ser submorfémicas: bajo el mismo morfema, más de una noción o contraste podía tener cabida, de manera que no era necesario dar una correspondencia de uno a uno entre contrastes gramaticales y contrastes morfofonológicos. Esta afirmación ha sido aceptada prácticamente de forma universal en las teorías morfológicas posteriores, que en cierto nivel de abstracción comparten la filosofía de esta visión, y ha sido interpretada como una forma directa de negar que los morfos cero puedan ser considerados objetos reales en el análisis de la palabra; como mucho, pueden tomarse como una convención formal útil para exponer procesos de formación a estudiantes, pero no se admiten como objetos del análisis morfológico real. Por ejemplo, Scalise (1983: 129-131) observa que un análisis del cambio categorial con morfos cero puede llevar a la necesidad de apilar morfo cero tras morfo cero en una misma palabra. Consideremos, por ejemplo, un ejemplo que Scalise no discute, pero que es relevante dentro de su filosofía: roto tomado como sustantivo (un roto en el pantalón) convertido a partir de un adjetivo que procede originalmente de un verbo romper. Siguiendo la lógica de Hockett (1947), la formación del participio verbal en ha roto debe implicar un morfo cero para el morfema de participio.

$$
\text { [[roto]V ø]Part-V }
$$

A continuación, el participio se convierte en adjetivo, y en este caso se emplea un segundo morfo cero.

$$
[[[\text { roto }] \mathrm{V} \quad \text { ø]Part-V } \quad \text { }] \mathrm{A}
$$

Y por último, el adjetivo se convierte a nombre con otro morfo cero.

$$
[[[[\text { roto }] \mathrm{V} \quad \varnothing] \text { Part-V } \quad \text { }] \mathrm{A} \quad \text { } \quad \text { N }
$$


Esto ha hecho que el análisis de la conversión categorial como sufijación cero haya sido rechazado mayoritariamente en favor de otras alternativas, como la existencia de entradas léxicas dobles (cf. Bauer y Valera 2005 para una revisión exhaustiva de la bibliografía sobre el tema). Esta situación es general en los estudios morfológicos. Frente al análisis de morfo cero, han surgido dos grandes familias de análisis que implementan técnicamente de formas distintas la propuesta de Nida. Por un lado, tenemos modelos de morfología amorfa en los que los casos en que no hay correspondencia entre forma y significado se interpretan como argumentos a favor de tratar la palabra, y no el morfema, como la unidad mínima de descomposición morfológica (Anderson 1992, Stump 2001); así, went sería la forma pasada de una palabra, y no cabría descomponerla en unidades menores. Por otra parte, tenemos una serie de teorías que proponen que es necesario tener representaciones distintas para la morfosintaxis y la morfofonología. Si bien los contrastes gramaticalmente relevantes en morfosintaxis implican la existencia de unidades discretas, no es cierto que las unidades morfofonológicas - los exponentes, en términos de Trommer (2012)- correspondan biunívocamente a ellas. De hecho, cabe esperar que un mismo exponente materialice simultáneamente dos o más unidades morfosintácticas, que es la forma en que se analizaría el caso de went en por ejemplo las modernas teorías nanosintácticas (Caha 2009).

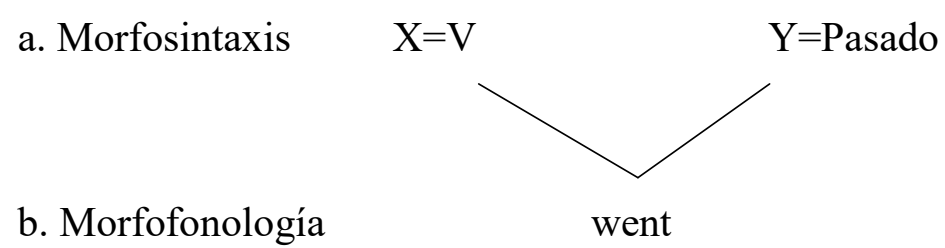

El análisis morfológico ha adoptado generalmente una de estas alternativas, en lugar de la postulación de morfos cero. Las escasas excepciones, como Myers (1984), emplean los morfos cero como objetos marcados cuyas condiciones de legitimación son muy restringidas: en el caso de Myers, deben ser siempre elementos terminales dentro de la secuencia de morfemas de una palabra.

No obstante, como nota Harbour (2008), este rechazo general a los morfos cero no tiene ninguna base real en la arquitectura de la gramática actual. No se conoce ningún principio morfosintáctico que fuerce a que la representación fonológica de un elemento terminal tenga que ser de un tipo determinado. De hecho, la evidencia que tenemos es 
que la sintaxis debe ser ciega a las condiciones puramente fonológicas (Pullum \& Zwicky 1988), de manera que la sintaxis no encuentra relevante si -por ejemplo- el sujeto de una oración debe comenzar por consonante o por vocal, o si debe tener una sílaba inicial con coda o sin ella. Resulta inesperado que la morfosintaxis haga una excepción precisamente con la representación fonológica de los morfemas, imponiéndoles el requisito de que no tengan una representación fonológica vacua. De hecho, en sintaxis se admiten generalmente los pronombres nulos, y no dejan de ser morfemas sin representación fonológica. Harbour concluye que, aunque postular un morfo cero no puede ser el punto de partida metodológico cuando se trata de analizar la estructura interna de una palabra, no hay ninguna motivación sólida para rechazarlos de entrada como posibles objetos del análisis morfológico.

El objetivo de este trabajo es precisamente el de argumentar que hay casos en los que es necesario postular un morfo cero, y que en tales casos hay pruebas empíricas de que estructuralmente ese morfo está presente en la estructura. Para entender la forma del argumento, veamos una representación como la de (9), frente a (10).

[[base] ø]

$$
\text { [base] }
$$

En la fonología, tendríamos la misma representación superficial, pero en morfofonología tenemos situaciones muy distintas. En (9) hay un morfo cero, que carece de representación fonológica, pero estructuralmente su presencia hace que la base no esté en el margen derecho de la palabra; en (10), en cambio, sí sucede esto. La diferencia es grande, y si realmente existe, esperamos que haya fenómenos que ayuden a discriminar entre los dos casos. En este trabajo vamos a argumentar que la supresión de la vocal temática con ciertos verbos es precisamente el tipo de fenómeno que permite argumentar a favor de la existencia de un morfema cero.

El trabajo se estructura de la siguiente manera. En $\S 2$ vamos a presentar los datos relevantes, que se relacionan con la desaparición de la vocal temática en el imperativo de verbos como salir o tener. Vamos a argumentar que esto es una operación morfofonológica, y no sintáctica. En $§ 3$ vamos a presentar el análisis del borrado, que crucialmente implica que el imperativo, siguiendo a Biezma (2009), carece de sintagma tiempo. En $\S 4$ vamos a mostrar que, dado lo anterior, es necesario postular que la 
tercera persona singular se expresa en español con un morfo cero. §5 presenta las conclusiones.

2. La vocal temática en el imperativo

En español todos los verbos de la primera conjugación forman el imperativo de la segunda persona singular de una forma muy simple: la raíz verbal y la vocal temática, sin otros morfemas adicionales.
a. cant-a
b. sueñ-a
c. mat-a
d. atac-a

La mayoría de los verbos de la segunda y tercera conjugación siguen el mismo patrón: nótese que el rechazo general a tener /i/ átona en posición final de palabra hace que la forma que emerja para la tercera sea $[\mathrm{e}]$.
a. viv-e
b. beb-e

No obstante, hay una serie delimitada de verbos en los que el imperativo de segunda persona es idéntico en la superficie a la raíz, es decir, en los que la [e] final desaparece. A esta clase pertenecen los siguientes verbos:

$$
\begin{aligned}
& \text { a. hacer }>\text { haz } \\
& \text { b. salir }>\text { sal } \\
& \text { c. tener > ten (y sus derivados: contener }>\text { contén }) \\
& \text { d. } \text { poner }>\text { pon (y sus derivados: oponer }>\text { opón }) \\
& \text { e. venir > ven } \\
& \text { f. yacer > yaz (desusado) }
\end{aligned}
$$

Los verbos que siguen esta regla comparten un aspecto de su representación fonológica: en todos los casos, la consonante es coronal, $/ \mathrm{r} /, / 1 / \mathrm{o} / \theta /$. Esto es solo un subconjunto de las consonantes que el español admite (patrimonialmente) en posición final de palabra (Harris 1983). Por ejemplo, si bien el español admite palabras terminadas en /d/, no hay 
casos de verbos cuya raíz termine en esta consonante que tengan un imperativo irregular.
a. pedir $>$ pid-e $($ no * pid $)$
b. agredir $>$ agred-e (no *agred)

Del mismo modo, no es cierto que todos los verbos cuya raíz termine en estas consonantes sufran este proceso de apócope de la vocal temática. Por ejemplo, terminados en $/ \theta /$ o $/ 1 /$ tenemos casos perfectamente regulares como los siguientes:

$$
\begin{aligned}
& \text { a. crecer }>\text { crec-e }(\text { no } * \text { crez }) \\
& \text { b. oler }>\text { huel-e (no *huel) } \\
& \text { c. pulir }>\text { pul-e (no *pul) }
\end{aligned}
$$

Por tanto, la regla que borra la /e/ final de la vocal temática debe estar léxicamente marcada, de forma que se aplica solo a una clase de verbos arbitrariamente elegidos de entre los que poseen ciertas propiedades fonológicas.

Podemos proponer una regla como la de (16), que es lo más neutral y tradicional posible -ya que nuestro análisis aspira a formar el argumento independientemente de la implementación técnica que se desee adoptar-. Las raíces están marcadas con un diacrítico arbitrario $\alpha$; el signo \# marca la posición final de palabra.

$$
\left.\mathrm{e}--->\varnothing / \mathrm{C}] \alpha_{-}\right] \#
$$

No obstante, esta regla aparentemente no captura correctamente los datos. La regla de (16) debería predecir que la conjugación de un verbo como tener en presente de indicativo debería ser la de (17).
a. teng-o
b. tien-e-s
c. $*_{t}(\mathrm{i}) \mathrm{en}$
d. ten-e-mos
e. ten-é-is
f. tien-e-n

La tercera persona singular debería emerger como el imperativo, ya que aparentemente la /e/ es final en esta palabra. Pero sabemos que esto no es el caso. 
Podría pensarse, inicialmente, que quizá la regla de (16) deba restringirse de forma que haga referencia expresa a que el verbo debe estar en una forma imperativa. No obstante, esto iría contra la modularidad estricta de la gramática: la representación de (16) hace referencia solo a objetos que son representaciones morfofonológicas del léxico: un conjunto de raíces acabadas en consonante que contienen, como ítems léxicos, un diacrítico, en las que la /e/ final de palabra se borra.Introducir la información de que además la regla se aplica a bases en imperativo es contradictorio con la separación modular entre sintaxis y fonología (cf. en este sentido Bermúdez-Otero 2012, para los problemas que surgen si se admite información puramente sintáctica o semántica en las representaciones morfofonológicas).

¿Qué sucede entonces para que la regla no se aplique a una forma como (17c)? Para responder a esta pregunta debemos primero examinar la estructura de los imperativos en español.

\section{Los imperativos}

Son bien conocidas las propuestas de que los imperativos se asocian a una estructura empobrecida en español y en otras lenguas (cf. Zanuttini 1997). Concretamente, se ha propuesto que los imperativos carecen de un nudo de Sintagma Tiempo, que en los marcos teóricos en los que se formula la propuesta es donde se localiza la concordancia entre el sujeto y el verbo. Aquí seguimos la propuesta concreta de Zanuttini (1997) y Biezma (2009).

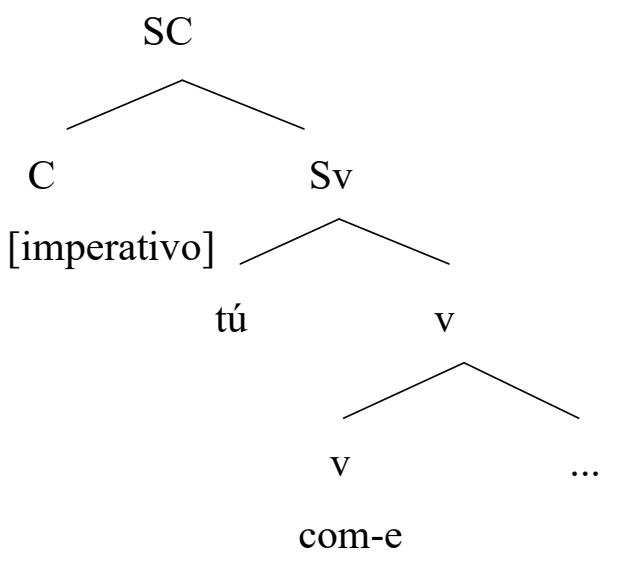


La propuesta general es que el verbo termina ascendiendo por movimiento de núcleo a C(omplementante), un núcleo que define la fuerza ilocutiva de la cláusula; allí coteja los rasgos de la modalidad oracional. Esto da lugar a una representación como la de (19):

$$
\left[[[\mathrm{com}-] \mathrm{e}]_{\mathrm{V}}\right]_{\mathrm{C}}
$$

Son varios los argumentos que apoyan esta estructura empobrecida para los imperativos, y que se han ofrecido en la bibliografía.

(a) Esta representación explica que el imperativo no admita negación (*No comed). En la suposición de que la polaridad negativa que niega la cláusula se localiza en una proyección que domina a ST (Laka 1990), esta propiedad se sigue del hecho de que la forma imperativa carece de $\mathrm{T}$ y por tanto un núcleo que seleccione $\mathrm{T}$ tampoco puede aparecer en la estructura.

(b) El inglés es una lengua en que los verbos deben aparecer necesariamente con un sujeto preverbal (20), lo cual se ha presentado como una propiedad especial del ST de estas lenguas (por ejemplo, véase Holmberg y Roberts 2009). Sin embargo, en el imperativo no aparece sujeto alguno (21).

$$
\begin{aligned}
& \text { *(you) eat. } \\
& \text { tú comes }
\end{aligned}
$$

Eat!

$$
\text { ‘Come!' }
$$

Esta propiedad se sigue si el ST está ausente de la representación de los imperativos positivos; al no estar presente $\mathrm{T}$, la propiedad que fuerza la presencia de sujetos explícitos en inglés desaparece al mismo tiempo.

(c) En español, el sujeto de los imperativos puede aparecer, pero necesariamente pospuesto (22). No puede pensarse que este sujeto esté localizado en ST, y que aparezca pospuesto porque el verbo ha terminado en C. De ser así, esperaríamos que el inglés también admitiera sujetos pospuestos con los imperativos (23), pero no es el caso.

$$
\text { Canta tú. }
$$

$$
\text { *Sing you }
$$


En cambio, si proponemos, como predicen las teorías de Zanuttini (1997) y Biezma (2009), que el imperativo carece de ST, el patrón se explica. El sujeto aparece en una posición baja, posiblemente dentro del sintagma verbal; esta es la misma posición que admiten los sujetos pospuestos en español. El inglés no admite sujetos posverbales, y por esta razón (24b) es tan agramatical como (23).

a. Ha venido Luis.

b. *Has come Luis.

(d) Los imperativos no admiten auxiliares de ningún tipo, lo cual es esperable si los auxiliares aparecen en el área por encima del Sv y esta área se encuentra radicalmente empobrecida en el imperativo.

$$
\begin{aligned}
& \text { a. }{ }^{*} \text { ¡Puede cantar! } \\
& \text { b. }{ }^{*} \text { ¡Ve a estar enfermo! } \\
& \text { c. }{ }^{*} \text { ¡Lleva dos horas corriendo! }
\end{aligned}
$$

Pues bien: si la estructura que se ha propuesto es correcta, predecimos precisamente que la representación morfofonológica del imperativo es la de (26), que equivale a (19) con la salvedad de que, al ser una representación morfofonológica, no son relevantes las etiquetas categoriales:

$$
[[[\mathrm{com}-] \mathrm{e}]]
$$

Por tanto, la [e] está en posición final de palabra, lo cual a través de la regla de (16) producirá la apócope de la vocal final para las raíces que, como elementos léxicos, lleven el diacrítico correspondiente:

$$
\text { sal-e ----> sal }
$$

\section{El presente de indicativo}

La diferencia morfosintáctica crucial con el presente de indicativo es que en esta forma sí se proyecta el ST, en el que se sitúan los morfemas de concordancia entre el sujeto y el verbo. 
(28)

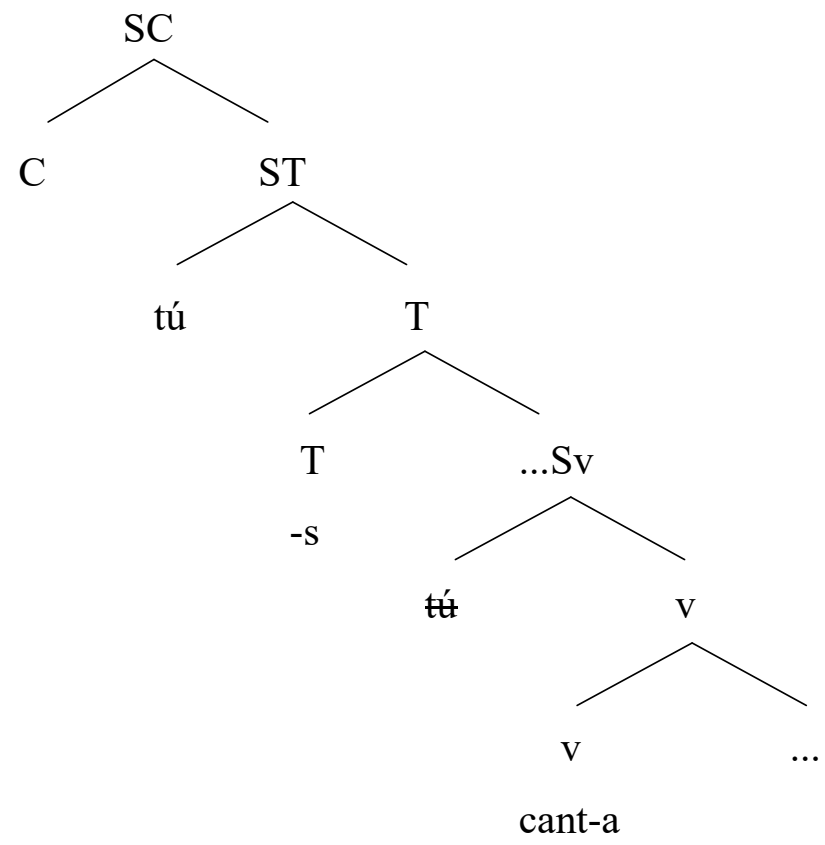

Por tanto, como sabemos, el inglés necesitará sujetos expresos en el presente, que además admite auxiliares y puede combinarse con la negación.

Todas las formas personales del presente tienen un segmento que puede asociarse morfofonológicamente con un valor para la persona y el número, salvo la tercera persona.

$$
\begin{aligned}
& \text { a. }-\mathrm{o}(1 \mathrm{sg}) \\
& \text { b. }-\mathrm{s}(2 \mathrm{sg}) \\
& \text { c. } ?(3 \mathrm{sg}) \\
& \text { d. }-\operatorname{mos}(1 \mathrm{pl}) \\
& \text { e. }- \text { is }(2 \mathrm{pl}) \\
& \text { f. }-\mathrm{n}(3 \mathrm{pl})
\end{aligned}
$$

Para la tercera persona caben dos alternativas. Quienes rechacen por principio la posibilidad de que haya morfos cero pueden proponer que en la tercera persona no existe marca morfológica alguna de concordancia. Esto no es imposible: puede suponerse que la interpretación por defecto de un tema verbal es de tercera persona singular. Esta propuesta podría estar apoyada por la idea de que la tercera persona es en realidad la ausencia de marca de persona (Benveniste 1966), y podemos ver el singular como el valor por defecto del sintagma de número, igualmente. De esta manera, en ausencia de morfemas que especifiquen los parámetros de persona y número, un tema 
verbal podría directamente interpretarse como los valores no marcados para las dos dimensiones de significado. No sería tampoco un problema que el mismo tema verbal se emplee en los imperativos, pero que en tales casos se interprete como segunda persona: los imperativos, por su fuerza ilocutiva, se orientan por defecto a un oyente. La propuesta no es en absoluto descabellada, y de ser así, la representación de una forma de presente de indicativo, tercera persona singular, sería la de (30) -suponiendo movimiento a $\mathrm{T}-$.

\section{$[[[\mathrm{com}] \mathrm{e}] \mathrm{V}] \mathrm{T}$}

Esto se traduce morfofonológicamente en la representación de (31), donde la /e/ vuelve a estar en posición final de palabra, como el imperativo.

$$
[[[\mathrm{com}] \mathrm{e}]]
$$

Pero entonces esperaríamos la forma sal, ten o pon también para la tercera persona singular del presente de indicativo, por la regla de (16).

Hay otros motivos para dudar de que esta teoría sea la correcta. ¿Es seguro que la interpretación por defecto de una persona, en ausencia de marcas gramaticales, deba ser la tercera? Cabe imaginar razones igualmente persuasivas para pensar que la lectura por defecto de un verbo emitido por un hablante deba ser la primera: a fin de cuentas, es el hablante quien ha decidido comunicarse con alguien para transmitirle información. Tampoco parece estar bien fundada la idea de que la forma de número no marcada deba ser el singular. De hecho, si deseamos preguntarle a alguien por un objeto del que no sabemos si hay uno, varios o ninguno, usamos el plural (32a) más que el singular (32b), que más bien empleamos para confirmar la información que creemos tener de que la persona tiene un hijo (cf. Grimm 2012).

$$
\begin{aligned}
& \text { a. ¿Tienes hijos? } \\
& \text { b. ¿Tienes un hijo? }
\end{aligned}
$$

Tampoco ayuda a la teoría de que la tercera persona singular se interpreta por defecto el hecho de que en inglés sea esta precisamente la forma que se marca de manera explícita.

$$
\begin{aligned}
& \text { sing-s } \\
& \text { canta-3sg }
\end{aligned}
$$

La única alternativa a la propuesta que se acaba de esbozar es precisamente la de que la 
tercera persona singular se expresa con un morfo cero en español, y que por tanto (34) es el paradigma de morfemas de concordancia de sujeto-verbo en presente:
a. $-\mathrm{o}(1 \mathrm{sg})$
b. $-\mathrm{s}(2 \mathrm{sg})$
c. $-\varnothing(3 \mathrm{sg})$
d. $-\operatorname{mos}(1 \mathrm{pl})$
e. - is $(2 \mathrm{pl})$
f. $-n(3 p l)$

Veamos cómo esto permite dar cuenta del contraste entre el imperativo y la tercera persona singular del presente. En presente, el verbo ascendería -como se considera estándar- al núcleo $\mathrm{T}$, donde tomaría la concordancia de tercera persona singular, un morfo cero.

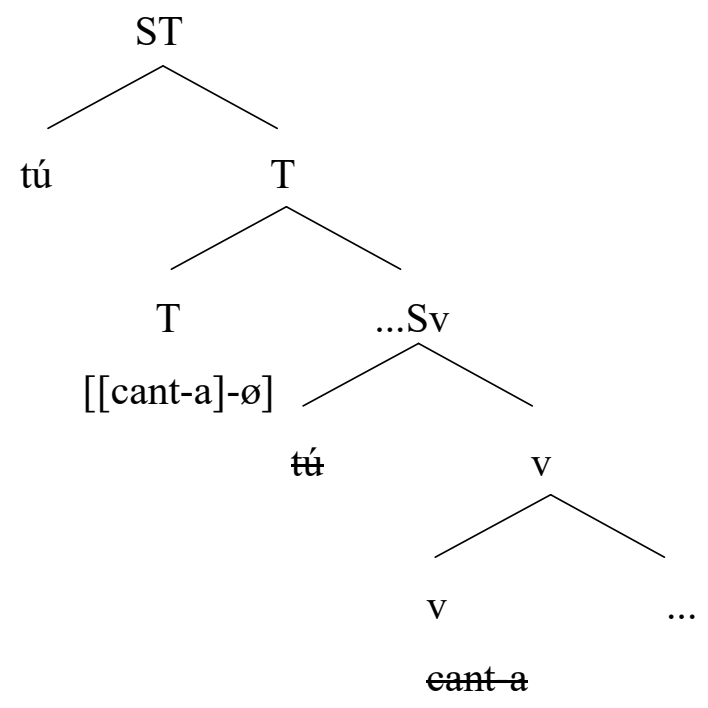

Ahora, la representación morfofonológica de la forma de tercera persona es la de (36):

$$
[[\text { cant-a]ø] }
$$

Esto implica que la representación de pon-e como tercera persona singular será la de (37).

$$
[[\text { pon-e }] \varnothing]
$$

Si bien la /e/ es el segmento final fonológicamente, morfofonológicamente no se encuentra en posición final de palabra, porque hay un exponente más entre el linde de palabra y él. (38) representa la diferencia entre el imperativo y el presente de indicativo a nivel morfofonológico. 

a. Imperativo: [[pon-e]]
b. Presente: [[pon-e]ø]

Lo crucial es que el exponente que contiene la /e/ no está en contacto con el linde de palabra en el segundo caso, pero sí en el primero. No es relevante si ese exponente tiene forma fonológica o no; eso le importa a la fonología, donde la diferencia entre las dos representaciones de (39) se disuelve. Pero en el nivel morfofonológico, esta diferencia implica que la regla de (16) se aplicará solo a la primera forma, no también a la segunda.
a. Imperativo --> pon
b. Presente --> pone

Por tanto, es necesario admitir que existe al menos un morfo cero en la morfología del español.

\section{Conclusiones}

En esta nota hemos propuesto que hay un argumento a favor de la existencia de al menos un morfo cero en español: si no admitimos que la representación de pone como forma de presente de indicativo es realmente [[pone- $] ø]$ la regla que apocopa el verbo en imperativo debería aplicarse también en este caso. La diferencia mínima entre las dos formas es sintáctica: el imperativo carece de $\mathrm{T}$ y por tanto de un exponente que materialice la concordancia de segunda persona singular, mientras que la tercera persona del presente introduce T. Si la gramática admite un morfo cero en este caso queda claro que no hay principios generales que impidan que estos objetos existan, por lo que no pueden ser rechazados de manera general en la teoría morfológica. Otra cuestión distinta es que no deban ser los primeros objetos en los que piense el investigador cuando trate de explicar un patrón de datos, pero esta es una cuestión metodológica que no afecta a los objetos que teóricamente admiten las lenguas naturales.

\section{Referencias}

Anderson, S. (1992).A-morphous morphology, Cambridge: Cambridge University Press. 
Bauer, L. \& S. Valera (2005).Approaches to Conversion / Zero-Derivation, Münster, Waxmann Verlag.

Benveniste, E. (1966). Problems in general linguistics, Coral Gables: University of Miami [traducción de 1971].

Bermúdez-Otero, R. (2012). 'The architecture of grammar and the division of labour in exponence', en J. Trommer (ed.), The morphology and phonology of exponence, Oxford: Oxford University Press, pp. 8-84.

Biezma, M. (2009). 'On the consequences of being small', en A. Schardl et al. (eds.), NELS 38, pp. 89-101.

Caha, P. (2009). The nanosyntax of case. Tesis doctoral, CASTL-Universidad de Tromsø.

Harris, J. W. (1983). Syllable structure and stress in Spanish, Cambridge (Mass.): MIT Press.

Hockett, C. F. (1947). 'Problems of morphemic analysis', Language, 23, 321-343.

Holmberg, A. (2010). 'Null subject parameters', en T. Biberauer et al. (eds.), Parametric variation : null subjects in minimalist theory, Cambridge: Cambridge University Press, pp.88-124

Laka, I. (1990). Negation in syntax: on the structure of functional categories and projections. Tesis doctoral, MIT.

Myers, S. (1984). 'Zero derivation and inflection', en M. Speas et al. (eds.), MIT WPL, Cambridge (Mass.): MIT Press, pp. 53-69.

Nida, E. (1948). ‘The identification of morphemes', Language, 24, 414-441.

Pullum, G. \& A. Zwicky (1988). 'The syntax-phonology interface', en F. J. Newmeyer (ed.), Linguistics: the Cambridge survey, Cambridge: Cambridge University Press, pp. 255-280.

Scalise, S. (1983).Generative Morphology, Dordrecht, Foris.

Stump, G. (2001). Inflectional Morphology: A Theory of Paradigm Structure, Cambridge: Cambridge University Press. 
Trommer, J. (2012). 'Ø-exponence', en J. Trommer (ed.), The morphology and phonology of exponence, Oxford: Oxford University Press, pp. 326-355.

Zanuttini, R. (1997). Negation and clausal structure, Oxford: Oxford University Press.

Recibido: 12 de agosto de 2016 Aceptado: 23 de noviembre de 2016 Publicado: 30 de noviembre de 2016 